\title{
MAYO CLINIC \\ Detection of Hypertrophic Cardiomyopathy Using a Convolutional Neural Network-Enabled 12-Lead Electrocardiogram
}

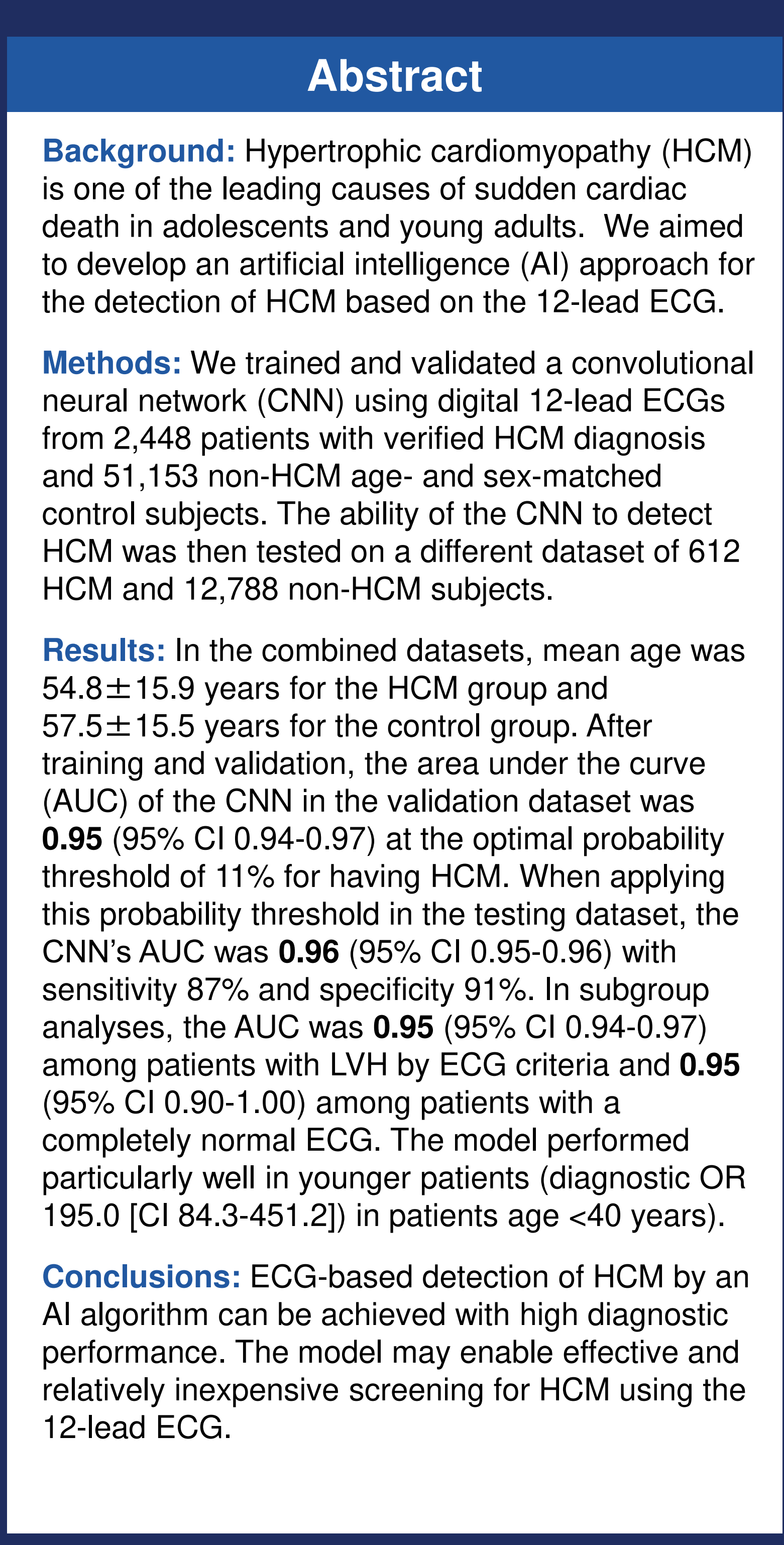

Wei-Yin Ko, MS, MEng*1; Konstantinos C. Siontis, MD*1; Zachi I. Attia, MS EE1; Rickey E. Carter, PhD2; Suraj Kapa, MD1; Steve R. Ommen, MD1; Steven J. Demuth3; Michael J. Ackerman, MD, PhD1; Bernard J. Gersh, MB, ChB DPhil1;
Adelaide M. Arruda-Olson, MD, PhD1; Jeffrey B. Geske, MD1; Samuel J. Asirvatham, MD1; Francisco Lopez--Jimenez, MD1; Rick A. Nishimura, MD1; Paul A. Friedman, MD1; Peter A. Noseworthy, MD1
Department of Cardiovascular Medicine, Mayo Clinic, Rochester, MN, Health Sciences Research, Mayo Clinic College of Medicine, Jacksonville, FL, Information Technology, Mayo Clinic, Rochester, MN

Wei-Yin Ko, MS, MEng*1; Konstantinos C. Siontis, MD*1; Zachi I. Attia, MS EE1; Rickey E. Carter, PhD2; Suraj Kapa, MD1; Steve R. Ommen, MD1; Steven J. Demuth3; Michael J. Ackerman, MD, PhD1; Bernard J. Gersh
Adelaide M. Arruda-Olson, MD, PhD1; Jeffrey B. Geske, MD1; Samuel J. Asivatham, MD1; Francisco Lopez--Jimenez, MD1; Rick A. Nishimura, MD1; Paul A. Friedman, MD1; Peter A. Noseworthy, MD1
Department of Cardiovascular Medicine, Mayo Clinic, Rochester, MN, Health Sciences Research, Mayo Clinic College of Medicine, Jacksonville, FL, Information Technology, Mayo Clinic, Rochester, MN

Mayo Clnic, Rochester, MN

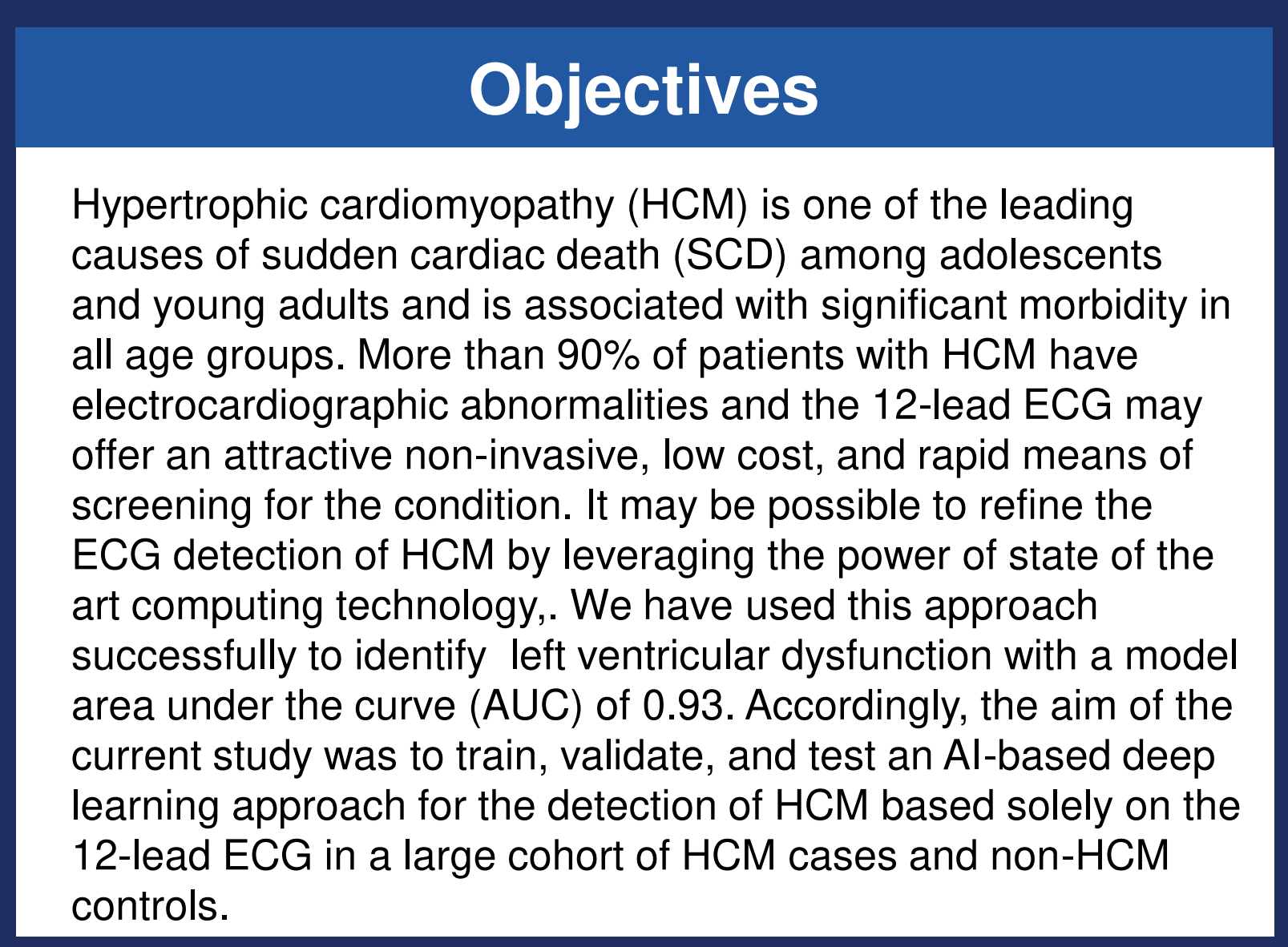

Figure 1: Model Performance

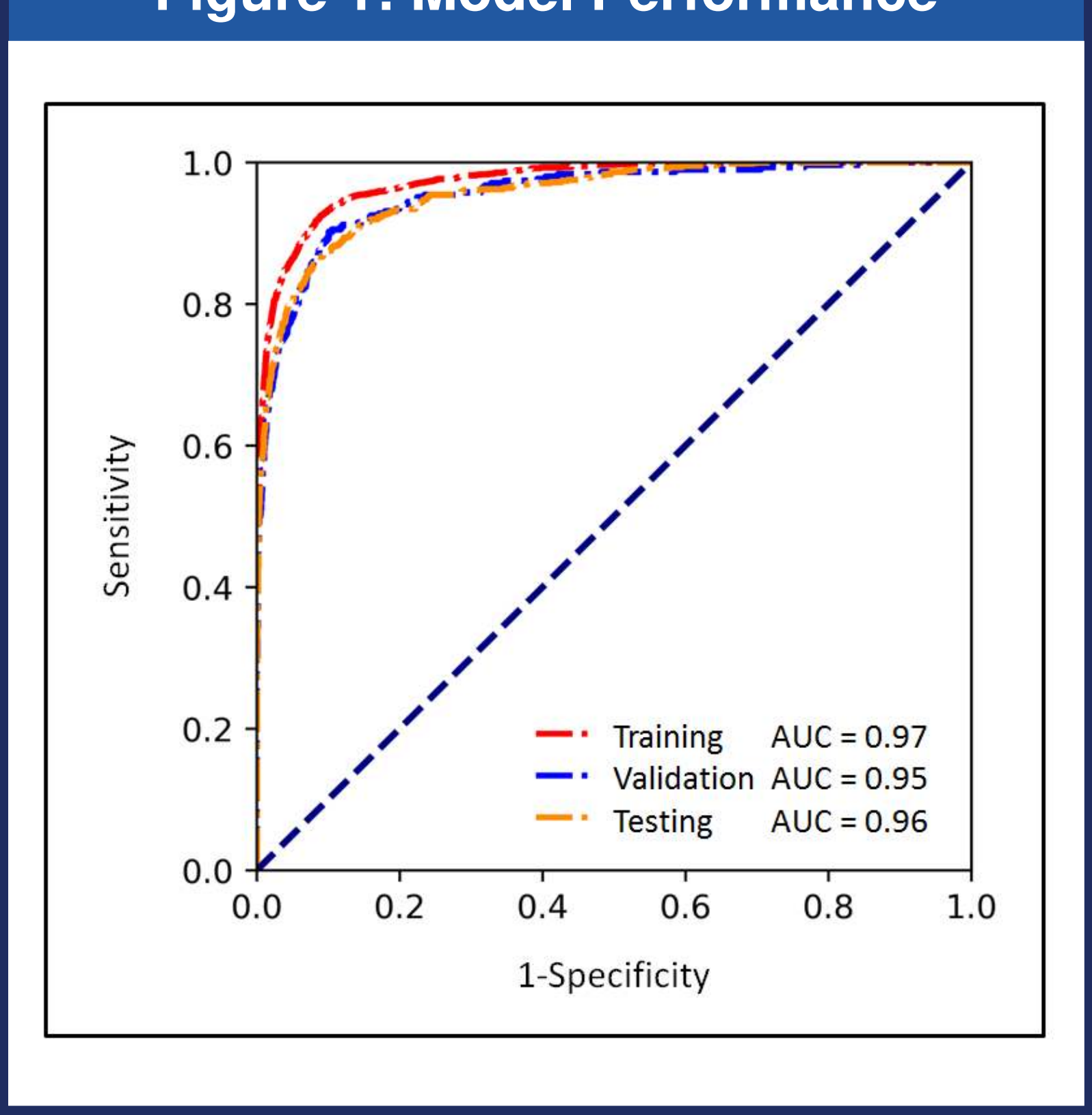

Results

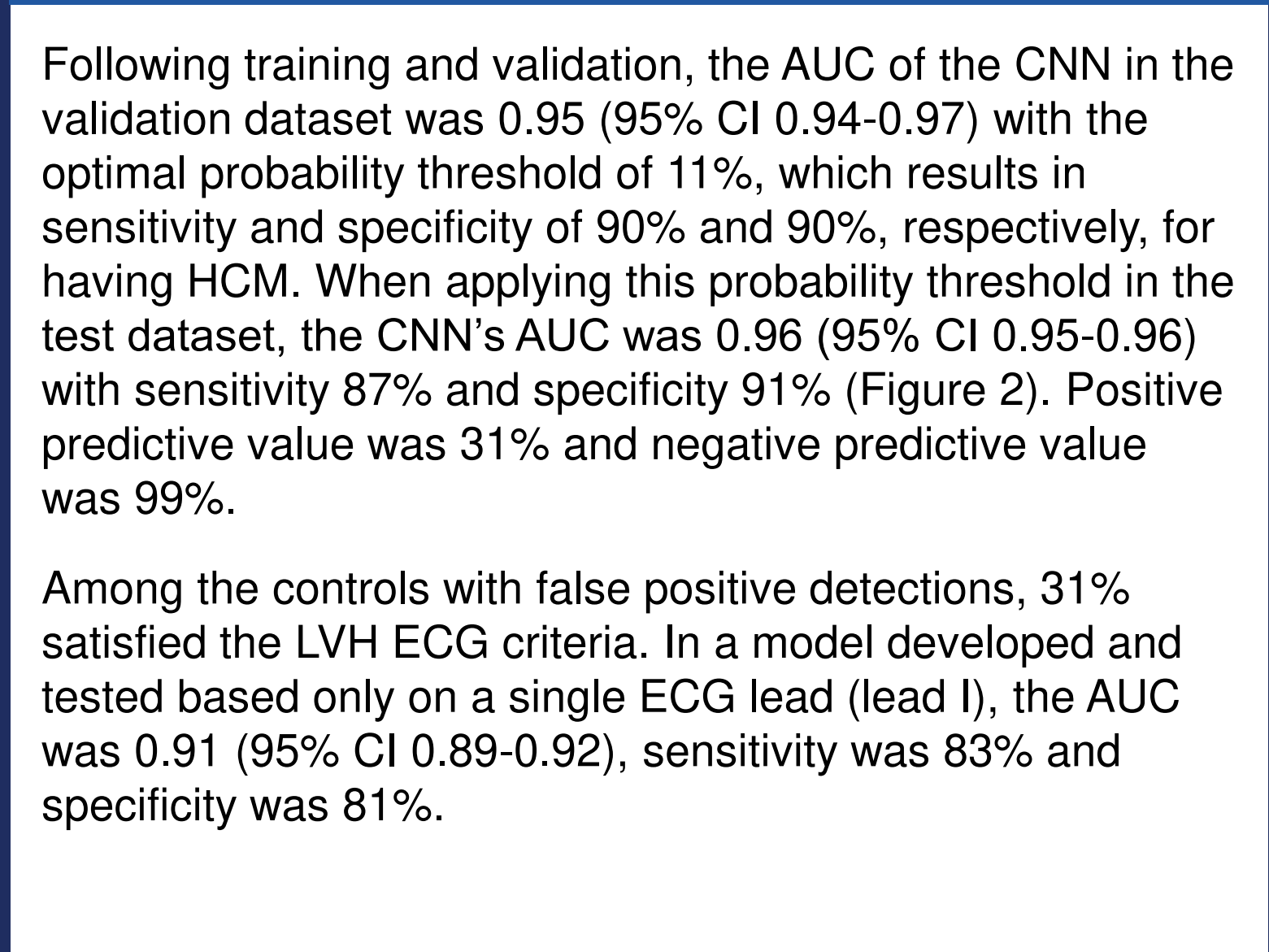

Methods

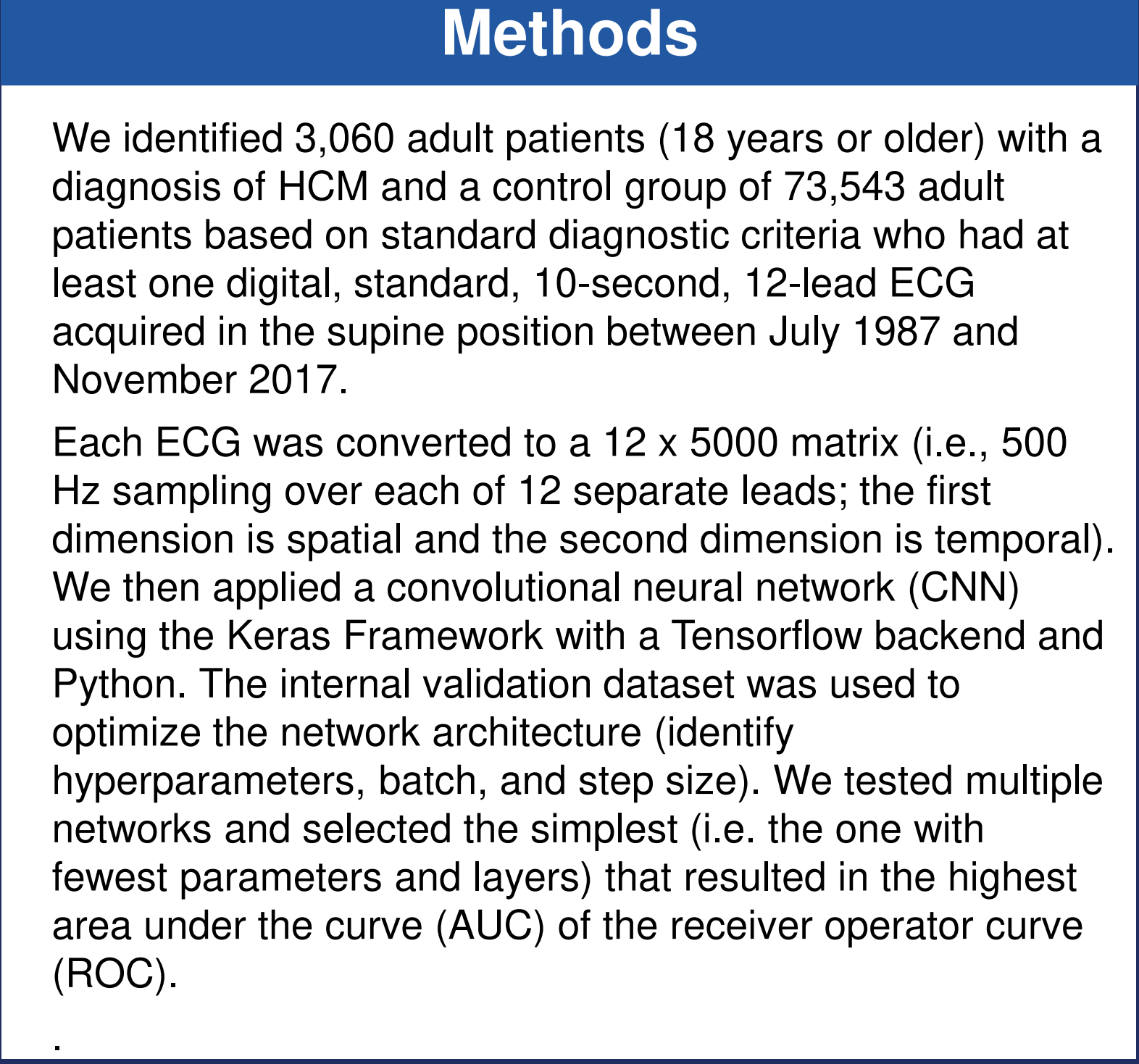

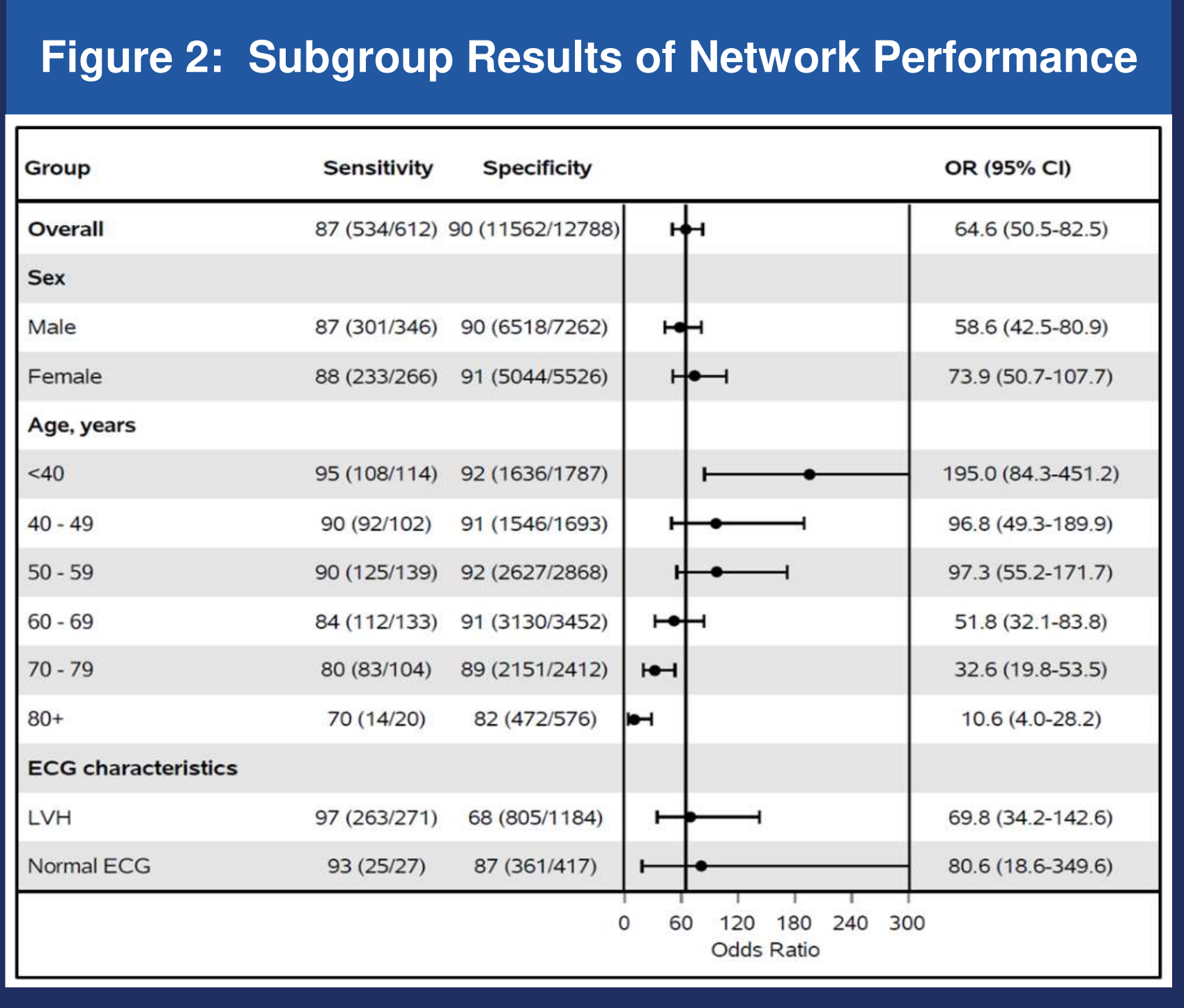

Figure 3: Model Development

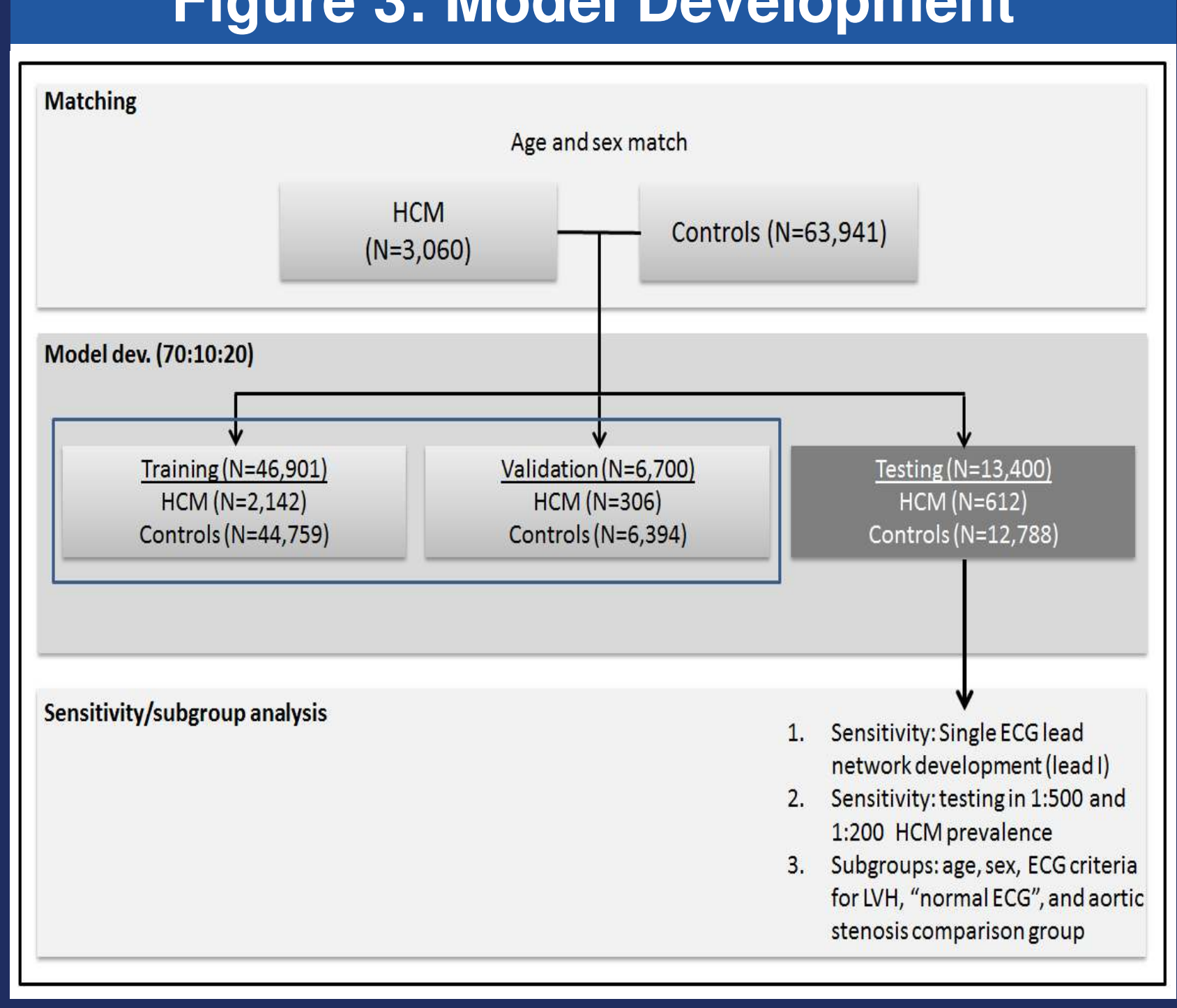

Discussion

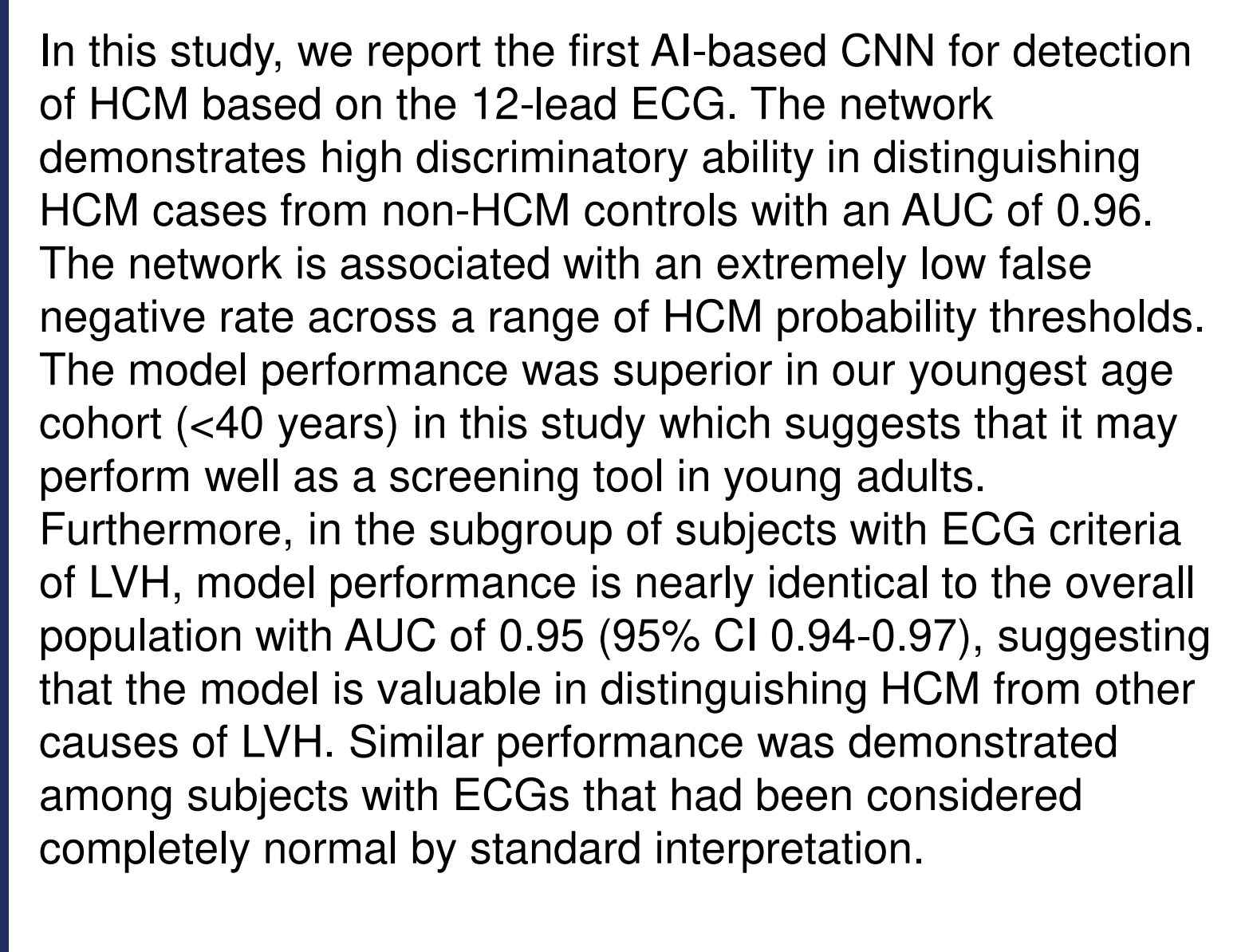

Conclusions

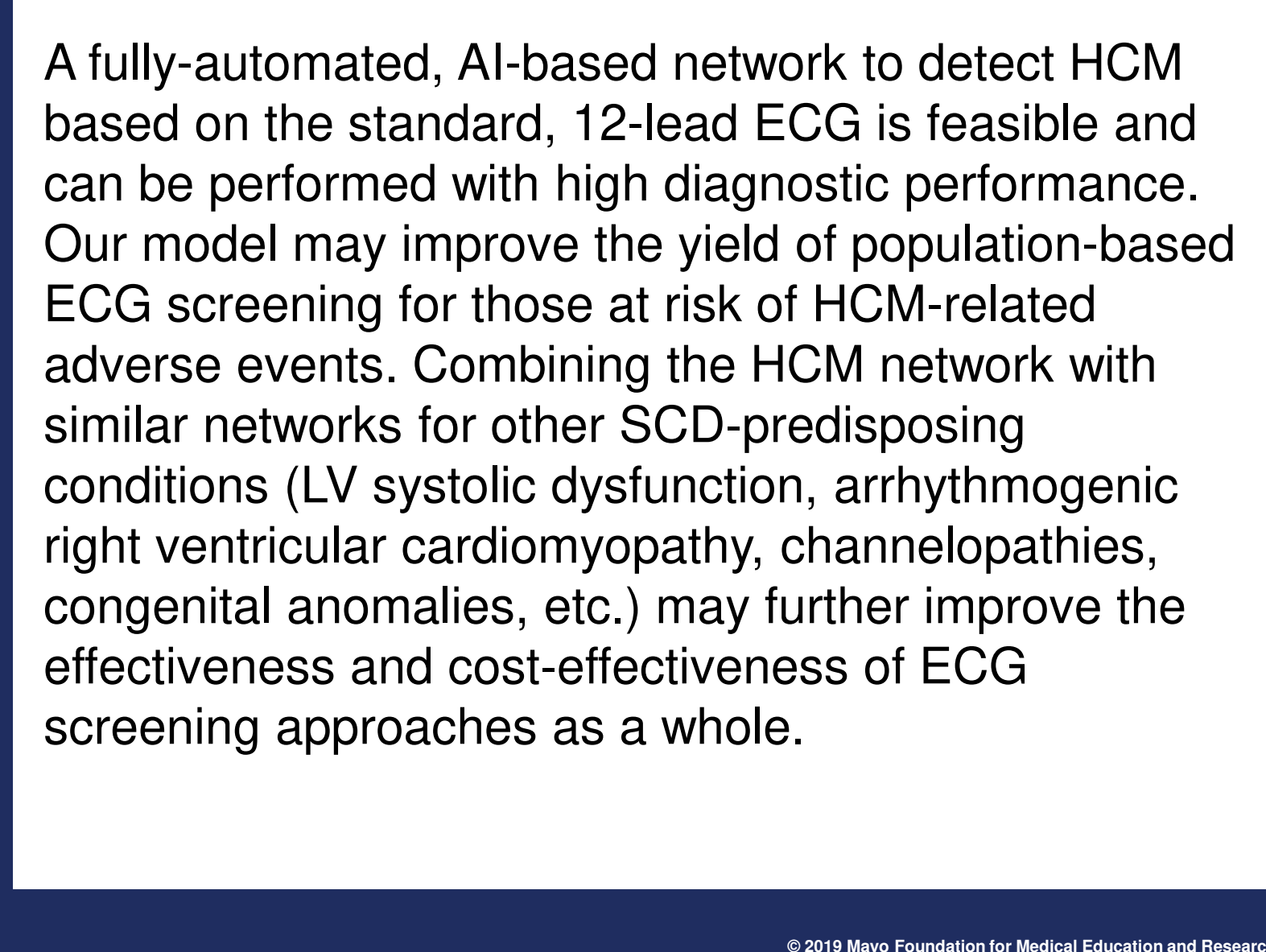

